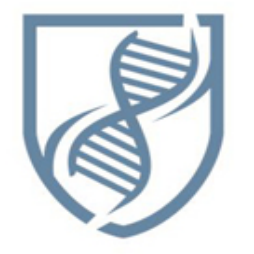

Journal of Bioscience and Applied Research

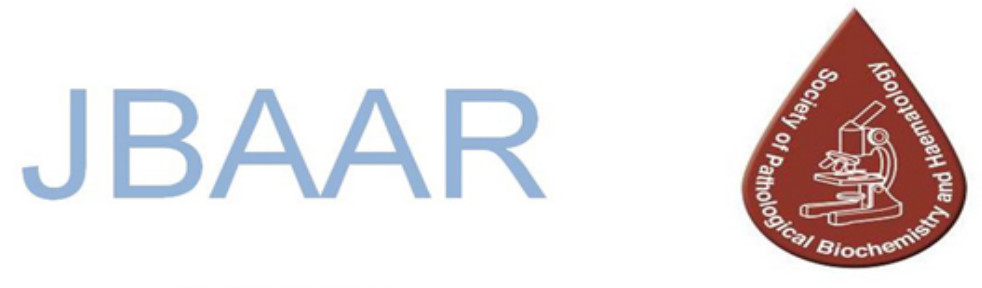

WWW.JBAAR.ORG

\title{
Effect of garlic on toluene-induced biochemical and histopathological effects in albino rats
}

\author{
Zuhair Y. A1-Sahhaf ${ }^{1}$, Osama M. Sarhan ${ }^{1,2}$ \\ ${ }^{1}$ Department of Biology, Faculty of Applied Sciences, Umm Al-Qura University, Makkah,Saudi Arabia. \\ ${ }^{2}$ Department of Zoology, Faculty of Science, Fayoum University, Fayoum, Egypt.
}

(Corresponding author e.mail: sarhanomm5975@gmail.com)

\begin{abstract}
The present study aims to study the effect of garlic extract on toluene inhalation at very low dose, induced hematological, biochemical and histological alterations in liver of albino rats. Animals were divided into 4 groups.Group 1 (G1) served ascontrols,G2 given garlic aqueous extract,G3 inhaled toluene vapor and G4 given garlic plusinhalation of toluene vapor. Animals were sacrificed after 2and 4weeks of treatment. The results showed that exposing animals to toluene induced significant decrease in red blood cell count (RBCs),hemoglobin (HGB),and blood platelets (PLT).On the other hand, the hematocrit percentage (HCT) and white blood cells(WBCs) count increased. Moreover, transaminases(ALT and AST) and gamma-glutamyl transferase (GGT) were increased in the sera of treated animals.Histological examination of liver of treated rats showed leukocytic infiltrations, congestion of blood vessels,cytoplasmic vacuolations of hepatocytes and fatty degeneration.Treated kidney in rats of G3 showed glomerular tufts congestion; renal space narrowing and epithelia of some renal tubules were degenerated with hemorrhage between them. To some extent, an improvement was observed in the kidney of the recovery group. Treating animals with garlic plus toluene caused an improvement in the biochemical and histological alterations in albino rats.It could be concluded that the protective effect of garlic may be attributed to the presence of organosulfur compounds which have antioxidant and detoxifying properties.
\end{abstract}

Keywords: Toluene, Garlic, Liver, Kidney, Rats, Hematology, Transaminases.

\section{Introduction}

Toluene is an industrial feedstock colorless hydrocarbon formed ofCH$_{3}$ (a methyl group)as attached side to a phenyl group, benzene ring. As a solvent, toluene and similar aromatic solvents are globally used in common household products as well aspetroleum and rubber industries (Niazet al., 2015).It can be produced either by catalytic conversion of petroleum or by aromatization of aliphatic hydrocarbons(Von Burg, 1993).In various industrial activities including shoe production and painting, workers who are constantly exposed to toluene and other organic solvents face health risksincluding hepatotoxicity, nephrotoxicity and hematotoxicity, lung cancer, damage of the blood forming organs, in addition, hazards in the reproductive and central nervous systems (Chang, et al., 2013; de Oliveira et al., 2011; Khan et al., 2013; Kim, 2010; Li et al., 2010; Low et al., 1988and Vaghasia et al., 2013; Zaman, et al., 2002,).Mostly, doses of toluene concentration were800 ppm, 2000-3000, 5000 ppm, and/or $10000 \mathrm{ppm}$, but at low concentration (800 ppm), as the lowest concentration of toluene tested, did not produce a significant effect (Amenoet al., 1992; Gerasimov et al., 2002 \&2003; Lee et al. (2006). Moreover, Amenoet al. (1992)reported that Wistar male rats were exposed to toluene vapor by inhalation (2000 or $10000 \mathrm{ppm}$ ) for $1 / 2 \mathrm{~h}$ or by oral administration (400 mg/kg) had significant changes in blood parameters. Toluenevapor exposure, at 600-800 ppm, induced confusion, auditory and visual hallucinations, inhibition, incoordination and nervous disorder, in addition, renal and hepatic damage (Brozosky and Winkler, 1965, Barbosa, 2006; Lindemann, 1991; Parafet al. 1993), Moreover, teratogenic effect, growth and skeletal retardation were noted in mice at lower exposure levels for at least half of the gestation period, (Hudak and Ungvary, 1978), as well as, decrease fetal weight and increase the frequency of cleft palate (Nawrot and Staples, 1979).Toluene and similar aromatic solvents gain their entrance through inhalation, intradermally or by inadvertent 
ingestion and distributed via systemic circulation fraction was used for the experiment at a dose of (Taofeeqet al., 2015) into vital organs including liver and $20 \mathrm{mg} / \mathrm{kg} / \mathrm{day}$ of garlic in $1 \mathrm{ml}$ saline.

kidney.

Garlic, Allium sativum (family Liliaceae),is one of the ancient medicinal plants that thas been cultivated as a food for over 5000 years. Ancient Egyptian records mentioned that use of garlic as a remedy for a variety of diseases(Block,1985).Garlic's active constituents include sulfur-containing compounds that are rapidly absorbed and metabolized (Bozin et al., 2008;Kodera et al., 2002), and rich in trace elements (Gorinsteinet al., 2005). The principal medicinal uses of garlic are to modulate blood pressure, cholesterol, fight infections, inhibits platelet aggregation, anti-thrombotic, antidiabetic, anti-solid tumorsand anticancer. Also, it has anti-inflammatory, hepatoprotective and antioxidant, as well as, other beneficial usesinclude antihelmentics, antifungal and wound healing (Jalali et al., 2009; Mikail, 2010, Londheet al., 2011, Bahramsoltani et al., 2014).Garlic extract was found to have a protective effect against hepatotoxicity of $\mathrm{CCl}_{4}$ (Wafayet al. 2012), ethanol (Hussein et al,. 2007) and dimethylnitrosamine (Shaarawyet al., 2009).

Studies carried out on garlic constituents have reported the presence of two main classes of antioxidant components,namely flavonoids and sulfur-containing compounds,diallyl sulfide,tri-sulfideandallyl-cysteine, (Bozin et al., 2008;Kodera et al., 2002).In addition to sulfur-containing compound,garlic is also rich in trace elements(Gorinsteinet al.,2005).The bulb of the plant has been used as a carminative,anti-septic,expectorant,antihelmintic and diuretic (Badieiet al. 2005).Administration of garlic has been shown to treat hyperlipidaemia in humans(Adler et al.,1997).Garlic extract was found to have a protective effect against hepatotoxicity of $\mathrm{CCl}_{4}$ (Wafayet al.,2012),ethanol(Hussein al,.2007)anddimethylnitrosamine(Shaarawyet al.,2009).

et

Due to the specific situation in the circulation,liver and kidneys are organs for xenobiotic transformation, chief regulator of body fluidsfor maintaining homeostasis, acidbase equilibrium of electrolytes in the body. However, after tolueneexposures and/or garlic extraction, the hematological profiles and histological alterations provide us important symptomsabout the status of internal environment of rats as mammalian model.Thus, the present study was designed to evaluate the potentially protective effect of garlic extraction, which maybe modulate alterations of hematological and biochemical parameters, as well as, hepatic and renal toxicity in albino rats exposed to extra low dose of toluene vapor.

\section{Materials and Methods Preparation of the garlic extract}

Garlic was obtained from local market at Makkah,KSA200 mg of fresh peeled garlic cloves were weighted by sensitive balance then homogenized with $10 \mathrm{ml}$ of distillated water to prepare a concentration of $20 \mathrm{mg} / \mathrm{ml}$. The homogenate was centrifuged at $3000 \times \mathrm{g}$ for $10 \mathrm{~min}$ to remove particulate matter and the supernatant

\section{Experimental design}

Sexually mature male albino rats weighing 150-170 g were used in the present study.All animals received professional humane care in compliance with the guidelines of the Ethical Committee of the Umm Al-Qura University,Saudi Arabia.They were housed in standard cages and left to acclimatize for one weekto laboratory condition before the commencement of the experiment. The animals were maintained on standard laboratory diet and water was availablead libitum. The rats were randomly distributed into four groups; 10 rats each.Rats of G1were served as control, G2 orally received $20 \mathrm{mg} / \mathrm{kg} / \mathrm{day}$ of garlic in $1 \mathrm{ml}$ saline for 4 weeks, G3 exposed to toluene vapor at a dose of $200 \mathrm{ppm} / 20 \mathrm{~min} /$ day for 4 weeks, G4 received garlic extract and after a hour they were exposed to toluene inhalation at a dose of $200 \mathrm{ppm} / 20 \mathrm{~min} /$ day for 4 weeks.

\section{Biochemical assays}

For hematological studies, two blood samples were taken by cardiac puncture from control and treated animals after 2 and 4 weeks of treatment for a complete blood count (CBC) and biochemical assays. CBC samples used to determine red blood cells count (RBCs), hemoglobin value (HGB), hematocrit value (HCT \%), white blood cells count (WBCs) and blood platelets (PLT) number were measured by a fully automated Coulter counter (Coulter Electronics Limited, England). The second blood samples were centrifuged at 5,000 rpm and stored and frozen at $-20^{\circ} \mathrm{C}$ until the time of biochemical analysis according to standard methods (Reitman and Frankel, 1975; Henry, 1974). Triglycerides, cholesterol, aspartate aminotransferase (AST),alanin aminotransferase (ALT) and Gammaglutamyl transferase (GGT) were measured using a fully automated Hitachi 911 analyzer (Tokyo, Japan). A commercial randox kits (Randox Laboratories, LTD, Ardomre, Crumlin, United Kingdom) were used in these analysis.

\section{Histological examination}

At the end of the experiment, overnight fasted animals (the control and experimental animals) were sacrificed and dissected under light ether anesthesia. Liver and kidneysamples were removed, cut into smallslices and immersed inneutral buffered formalin $10 \%$ for 24 h.Fixed tissues were processed routinely, embedded in paraffin, sectioned at 5 micrometers thickness, de-paraffinized rehydrated and were stained with haematoxylin and eosin using the standard techniques (Bancroftand Gamble, 2002).

\section{Statistical analysis}

The results were expressed as mean \pm SD of different groups.The differences between the mean values 
were evaluated by ANOVA. Data were analyzed using the computer program SPSS/ version 15.

\section{Results \\ Biochemical results}

Results in figure(1)showed that the number of erythrocytes was significantly decreased in sera of rats after one and two treatments with toluene. On the other hand,a significant increase in total RBCs was recorded in animals given toluene andgarlic.Similarly, hemoglobin content was significantly decreased after one and two weeks of exposing to toluene.The hemoglobin content increased after treatment with toluene and garlic(Fig. 2). The hematocrit percentage was significantly increased after exposing rats to toluene, and decreased after treatment with toluene and garlic(Fig. 3).The number of platelets showed a significant decrease in toluene-treated rats and increased after treatment with toluene and garlic(fig. 4).On the other hand,the leucocyte counts were found to besignificantly increased after 2 weeks of treatment with toluene.The WBCs decreased after treatment with toluene and garlic (Fig. 5).No changes were recorded in blood parameters of control and garlic treated groups.

Concerning the change in liver function enzymes,data in figures(6-8)showed that values of ALT,AST and GGT increased significantly in rats exposing to toluene for two and four weeks.On the other hand,animals of G4 given toluene plus garlic extraction revealed significant decrease in mean values of these enzymes.

\section{Histological results}

Liver of rats in G1 showed normal histological profile, the hepatic cells were found arranged in strands around the centralvein,in cords intermingled by blood sinusoids containingnormal kupffer cells (figs. 9a \& b).Also, liver obtained from rats of group 2 treated with garlic extract exhibited the normal structure(Fig. 9c). In the liver of G3,exposed to toluene vapor,the hepatic lobule dilated central vein, the hepatocytes lost their normal architecture, their cytoplasm containing numerous vacuolations and fatty infiltration, some of them were degenerated with pyknotic nuclei, in addition, the sinusoids contain diffuse kupffer cells proliferation (figs. 9d-f). In the liver of the recovery group (G4), dilated sinusoids and hypertrophied kupffer cells can be seen occasionally, while in other liver tissues showed nearly restoring of their normal cytoarchitecture (figs. 9g, h).Kidney of rats in G1 and 2 showed normal cytoarchitecture of Glomeruli, renal tubules, renal spaces (figs. 10a \&b). In G3, glomerular tufts were congested with narrowing renal spaces, and the renal tubules showed degenerated epithelia with hemorrhage between them (fig. 10c). In the recovery group (G4), renal profile is nearly restoring its normal structure.

\section{Discussion}

The present results showed that exposing rats to toluene caused different biochemical changes.Inhalation of toluene vapor at low dose(200 ppm) showed noticeable adverse effect to the internal environment including hematological, biochemical and histological alterations, in contrary, the lowest concentration of toluene tested, 800 ppm, did not produce a significant effects (Gerasimovet al., 2003). Concerning the hematological changes, RBCs, hemoglobin and blood platelets contents decreased while hematocrit percentage (HCT\%) and WBCs increased. However, khan et al. (2013) studied the effects of some hazardous materials during shoe making on biochemical and hematological parameters. They reported normal blood glucose and hemoglobin levels, total red blood cells count, hematocrit value, mean corpuscular volume, mean cell hemoglobin level, mean cell hemoglobin concentration, while a significant decrease reported in platelets and neutrophil counts. In contrast, they recorded a significant increase in total leukocyte count, lymphocytosis, Eosinophilia and monocyte count, blood triglyceride, cholesterol, low density lipoprotein, high density lipoprotein, serum glutamate pyruvate transaminase, alkaline phosphatase, creatinine, and uric acid level of shoe workers. Our data in agreement with these results, Moszczyński and Lisiewicz(1983\& 1985)studied the hematological indices of peripheral blood in 121 workers occupationally exposed to benzene,toluene and xylene.They found that the first hematological changes noted in the workers were diminishing the mean corpuscular hemoglobin,the mean corpuscular hemoglobin concentration in erythrocytes andincreased numbers of reticulocytes,lowered total count of leukocytes due to decreased numbers of $\mathrm{T}$ and "non-T,non-B" cells as well as increased numbers of monocytes.

Transaminases(ALT and AST)and gamma-glutamyl transferase (GGT) were increased in the sera of treated animals.Ayanet al. (2012)investigated the acute toxic effects of high-dose toluene and its mechanisms on the liver tissue of toluene-treated rats.The level of plasma transaminase was found to be increased in toluene administered rats.Taset al. (2011)mentioned the harmful effects of toluene inhalation in the liver of rats and possible protective effects of melatonin on these detrimental effects.Toluene inhalation significantly increased serum ALT and AST,and decreased serum albumin,but did not affect serum ALP and total bilirubin levels.Kinghtet al.(1991)reported an increased level of serum transaminases and gama-glutamyltranspeptidase(GGT)in workers in the printing industry exposed to toluene via the respiratory route.

Examination of liver of toluene-treated rats showed many histopathological alterations include degenerating hepatocytes, aggregation of inflammatory cells infiltration, congested blood sinusoidswith hypertrophied kupffer cells in the hepatic lobules, in addition, dilatation and congestion in the bile ducts, portal veins and some portal venuoles, oedema, with active fibroblasts in the portal region.Also, Sarhan and Hussein (2016), found that liver of rats, exposed to isopropanol, showed degenerated hepatocytes with some pyknotic nuclei, degenerated kupffer cells, and the portal veins are dilated and congested.Similarly,some investigators (Ann et al., 2001and Hussein et al.,2008)studied the workers exposed to organic solvents 
and detected increased levels of liver transaminases and GGT, which indicates hepatic necrosis and cholestasis.One study of eight print workers occupationally exposed to low levels of toluene $\left(\leq 200 \mathrm{ppm}\right.$ or $\left.754 \mathrm{mg} / \mathrm{m}^{3}\right)$ reported mild elevation of serum transaminases including aspartate aminotransferase with concomitant pericentral fatty changes in the liver,as measured by liver biopsy(Guzelian et al.,1988). It was reported that ethylbenzene administration in male and female rats by oral gavage induced histopathological and serum chemistry changes(Mellertet al., 2007).At 13-week exposure,serum chemistry changes included increased alanine aminotransferase, total bilirubin, cholesterol, potassium, calcium and magnesium levels were recorded. Increased incidences of hepatocyte centrilobular hypertrophy and hyaline droplet nephropathy were observed after 4 and 13 weeks of exposure. Gonzalez-Yebra et al. (2006) documented that toluene may be a factor associated with the presence of renal damage in exposed shoe workers. Also, Taofeeq et al. (2015) concluded that exposure to different organic solvents have been reported to cause adverse effects on the hematological and functional integrity of different tissues in the biological systems. The same authors reported significant increase in ALT, AST transaminases and conjugated bilirubin, while total white blood cell count (WBC) was significantly decreased.

As regards to liver fibrosis reported in previous publications, the present work confirmed the presence of active fibroblasts, which represent an early stage for liver fibrosis. Moreover, the initiation of hepatic fibrosis may be due to stimulating glucocorticoid production, as well as the expression of glucocorticoid receptors and leptin receptors, which mediate the development of hepatic toxicity (Gotohdaet al., 2009; Wynn et al., 2006).Taofeeq et al. (2015) found that values obtained for total bilirubin (TB), conjugated bilirubin (CB), alanine transaminase (ALT) and aspartate transaminase (AST) were significantly higher (P0.05) in all other liver function parameters between the exposed painters and non-exposed control. Kidney function profiles showed no significant difference $(\mathrm{P}>0.05)$ between the exposed painters and non-exposed control. Hematological profiles revealed no significant difference $(\mathrm{P}>0.05)$ in packed cell volume (PCV) between the exposed painters and non-exposed control, total white blood cell count (WBC) was significantly lower.

As regards to kidney alterations observed in the present work, Taofeek et al. (2015) studied the effects of organic solvents on samples liver, kidney and bone marrow function parameters of 75 volunteers (Nigerianmalevehicle spray painters). They proved that these solvents induced nephrotoxicity, hepatotoxicity and haematotoxicity. Moreover, Sarhan and Hussein (2016) reported that rats exposed to isopropanol vapor showed increase in the cellularity of the glomerular tufts, degenerated epithelia of some renal tubules that contain colloid discharge, and hemorrhage between them as well as diffused hemorrhage in some renal spaces.While, Killeen et al.(2011) reported that isopropyl ingestion accidentally may cause little metabolic or renal abnormalities, false elevation of serum creatinine.

Treating rats with garlic extract was found to ameliorate the toxicity of toluene recorded in this work.Animals treated with garlic and toluene showed normal blood parameters.Similarly,Sharmaet al. (2010)reported that chronic lead nitrate ingestion showed a significant decline in total erythrocyte count,total leukocyte count,hemoglobin concentration,lymphocyte and monocyte content,while neutrophil content increased.On the other hand,treatment with garlic attenuated the deranged parameters to some extent. Toluene led to increase in liver enzymes ALT, AST and GGT, and caused liver histopathological alterations, while treatment with garlic reduced the activity of these enzymes and prevent the histopathological alterations. Serum AST,ALT and GGT are biomarkers in the diagnosis of hepatic damage because they are released into the circulation after cellular damage. It was reported that garlic extract preventsliver damage and reduced serum transaminases in rats treated with $\mathrm{CCl}_{4}$ (Wafay et al.,2012)and dimethyl-nitrosamine (Shaarawy et al.,2009).Garlic extract has been shown to decrease liver enzymes in serum and prevent liver damage of rats with liver fibrosis(Gedik et al.,2005).

The present results showed that administration of garlic with toluene resulted in improvement of hepatotoxicity of toluene.This could be attributed to the role of garlic as an antioxidant that preventing the accumulation of toxins within the cell.These findings are in agreement withthe study of many investigators who proved the antioxidant effects of garlic.Mirunalini et al.(2004) reported that garlic exerts its chemopreventive effects by modulating lipid peroxidation and enhancing antioxidant status in the liver and blood during buccal pouch carcinogenesis induced by 7 , 12dimethylbenz[a]anthracene (DMBA) in male Syrian hamsters.Bedi and priyanka (2012) showed that garlicdecreasedDMBA- induced increased oxidative stress through the significant decrease in lipid peroxidation and significant increase in the antioxidant enzymes,SOD and CAT.Administration of garlic can modulate the oxidative stress and improve the antioxidant system through the direct cytoprotective effect of garlic oil constituents;diallyldisulphide(DADS,60\%),allyl-

propyldisulphide(6\%), allicin(0. 3-0. 5\%) and diallyltrisulphide (DATS) (Hassan et al., 2010;Pariet al.,2007).Thus, the hepatoprotective effect of garlic observed in the present work may be attributed to the presence of organosulfur compounds which have antioxidant and detoxifying properties.

\section{Conclusion}

The obtained results concluded that low dose of toluene vapor induced significant decrease in RBCs, HGB, and PLT, meanwhile the HCT percentage and WBCs count increased. Moreover, transaminases (ALT and AST) and gamma-glutamyl transferase (GGT) were increased in the sera of treated rats in G3. Histological examination of liver of rats treated with toluene vaporG3 showed leukocytic infiltrations, congestion of blood vessels, cytoplasmic 


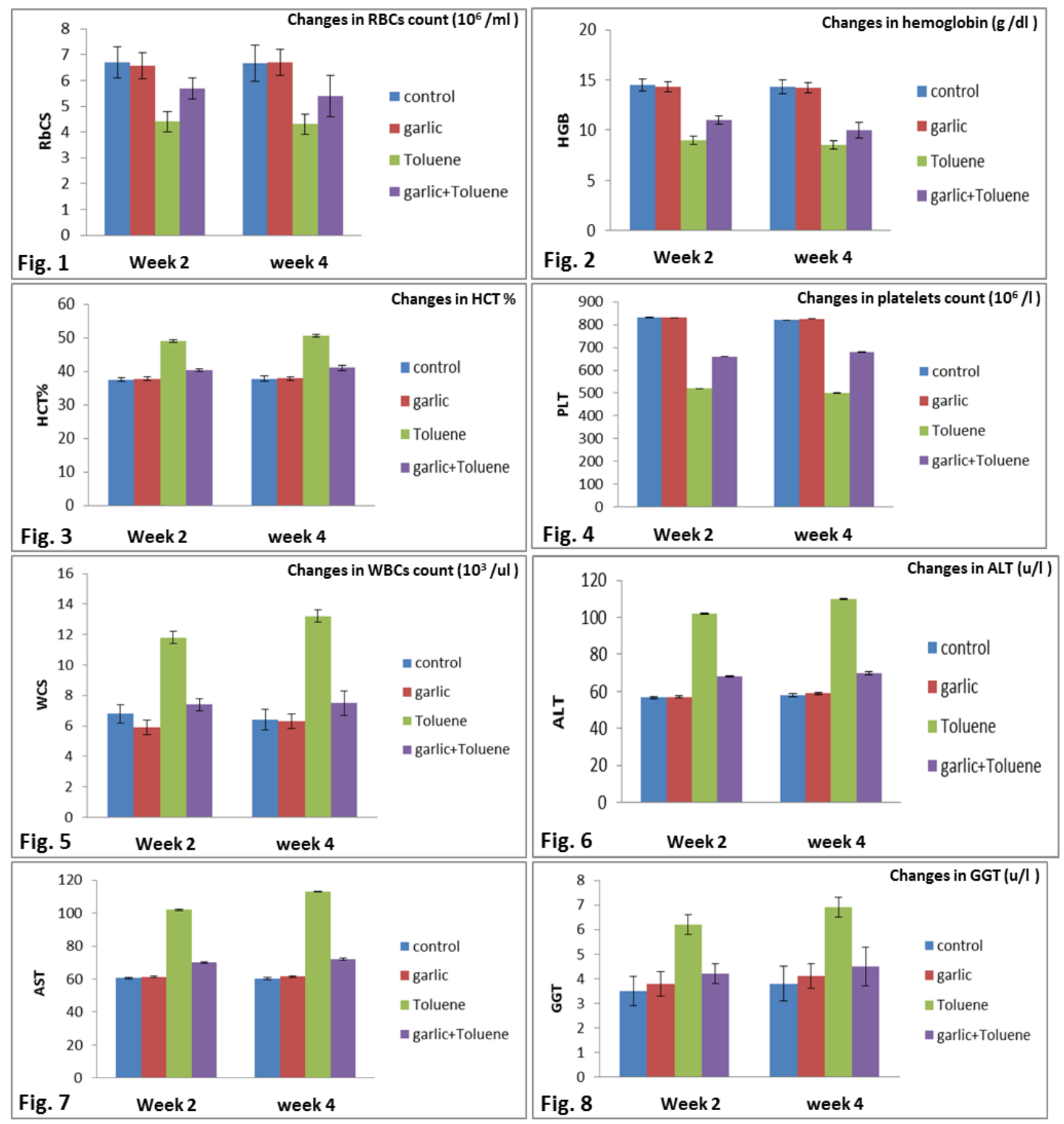

Figures 1-8: showed changes in haematological and biochemical parameters in the different animal groups. Fig. 1: RBCs $\operatorname{count}\left(10^{6} / \mathrm{ml}\right)$, Fig. 2: hemoglobin (g/dl), Fig. 3: HCT\%, Fig. 4: platelets count $\left(10^{6} / \mathrm{l}\right)$, Fig. 5: WBCs $\left(10^{3} / \mathrm{ul}\right)$, Fig. 6 : $\operatorname{ALT}(u / l)$, Fig. 7: AST (u/l), Fig. 8: Changes in GGT (u/l). 


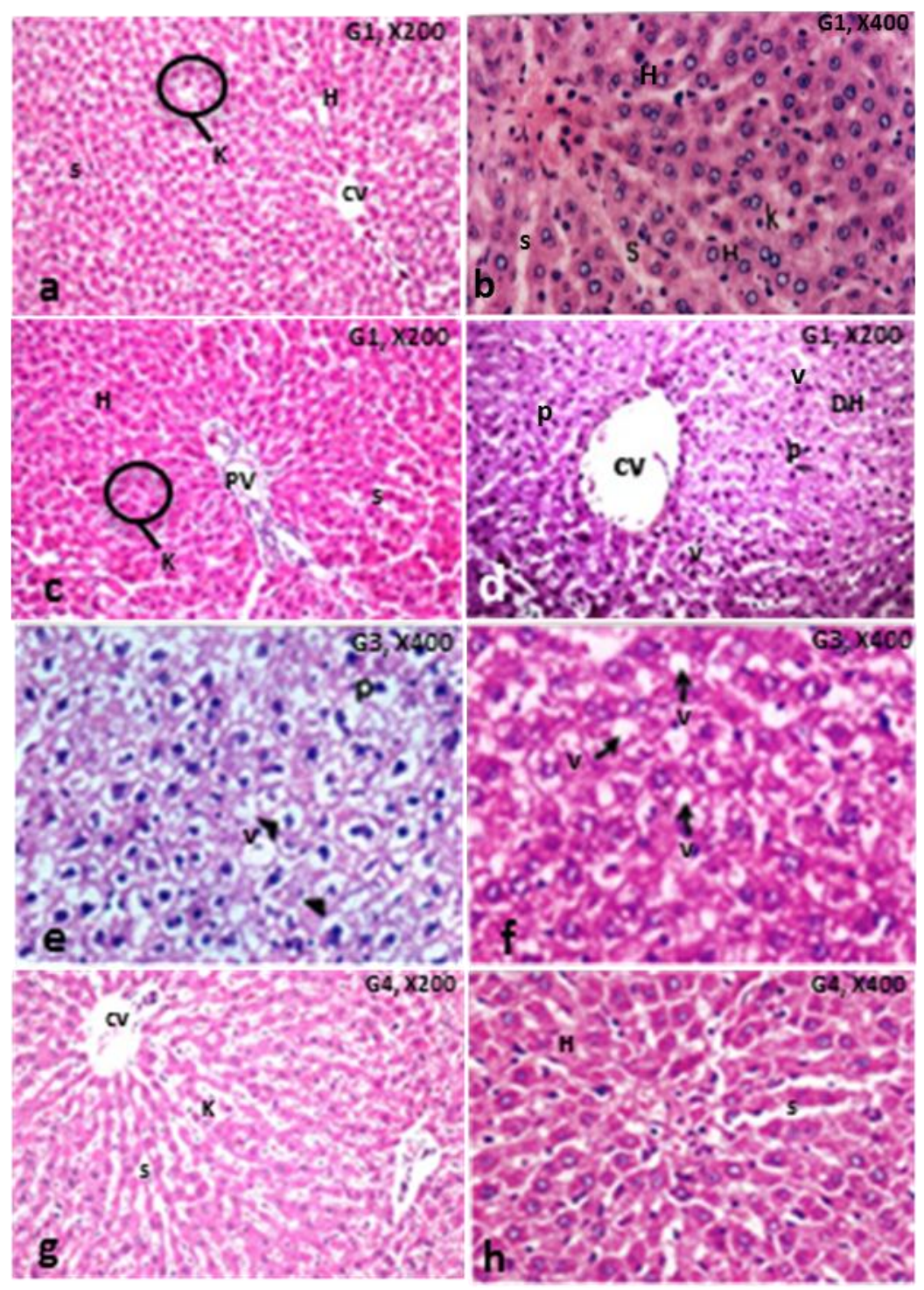

Fig. 9a\&b: Liver of control rats in G1 showed normal hepatic lobule, which consists of central vein (CV), cords of normal hepatocytes (H) surrounded by blood sinusoids (S), which contain normal kupffer cells. H\&E. X 200.

Fig. 9c: Liver of control rats in G2, treated with garlic extraction, showed normal portal area that includes hepatic artery (HA), portal vein (PV), and bile duct (BD). H: hepatic cells. H\&E. X 400.

Fig. 9d-f: Liver of rats in G3 showed dilated central vein (CV), cytoplasmic vacuolations (V), fatty infiltration (f), degeneration in the hepatocytes (DH) with pyknotic nuclei and the blood sinusoids illustrated diffuse Kupffer cells proliferation (K). H\&E.

Fig. 9g-f: Liver of rats in G4 treated with toluene plus garlic extraction showed acceptable improvement in the liver tissue. In figure 9g the hepatic parenchyma showed dilatation in the central vein, congestion in the hepatic sinusoids (S) with hypertrophy in some kupffer cells (K). In some rats of G4, the hepatic profile showed normal hepatocytes and blood sinusoids.H\&E. 


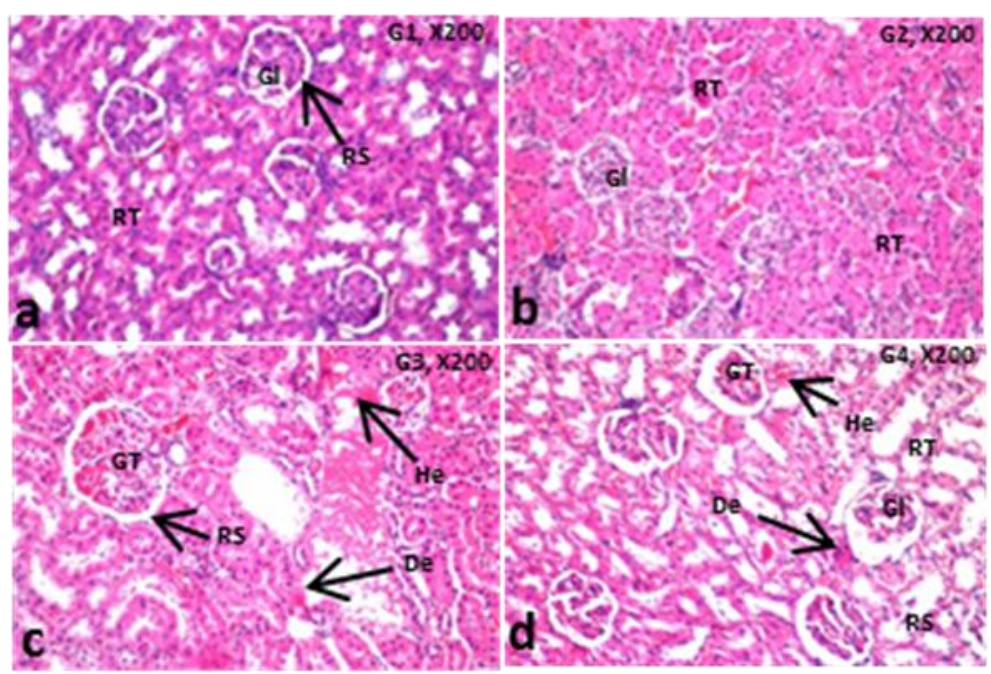

Fig. 10a: Renal cortex of control rats in G1 showed normal architecture of renal glomeruli (Gl), renal spaces (RS) and renal tubules (RT). H\&E. X 40.

Fig. 10b: light micrograph in kidney of G2 treated with garlic extraction showed normal histological structure of renal cortex. Gl: glomerulus, RS: renal space, RT: renal tubules. H\&E. X 40.

Fig. 10c: Kidney of rat in G3 showed narrowing renal space (RS), congestion in some glomerular tufts (GT), congested blood vessels (BV), degenerated epithelium (DE) of some renal tubules with few focal hemorrhage (He) between them. H\&E. X 40.

Fig. 10d: Histo-profile of Kidney in G4 treated with toluene plus garlic extractions showed improvement in the renal cortex, dilatation in some renal space, shrunken glomerular tufts (GT) and few focal hemorrhage (He) in between some renal tubules (RT). H\&E. X 40.

vacuolations of hepatocytes and fatty degeneration. Also, fumes of this solvent and its analogs of other organic kidney in G3, showed congested glomerular tufts, solvents. Noticeable improvement was observed in the narrowing renal space and degenerated epithelia of some recovery group including the biochemical and histological renal tubules with hemorrhage between them. As regards to alterations in albino rats. The gainedprotective effect of dose of toluene vapor used, the present authors concluded garlic may be attributed to the presence of organosulfur that inhalation of toluene vapor at low dose has adverse compounds which have antioxidant and detoxifying effect, which leads us to recommend avoiding the sniffing properties.

\section{References}

Adler AJ andHolub BJ(1997).Effect of garlic and fish oil supplementation on serum lipid and lipoprotein concentrations in hypercholesterolemic men.Ame J ClinNutr., 65: 445-50

Ameno K, Kiriu T, Fuke C, Ameno S, Shinohara T and IwaoIjiri I (1992). Regional brain distribution of toluene in rats and in a human autopsy. Arch Toxicol., 66: 153- 156.

AnnC,LeeK,ParkJ,JangJ,and KimM (2001).The association of exposure to organic solvents with liver function.Korean Journal of Occupational Environmental Medicine,13:64-74.

AyanM,TasU,SogutE,KulogluT,CayliS,KocamanN, KaracaZI,SahinM(2012).The apoptotic effect of a high dose of toluene on liver tissue during the acute phase,an experimental study.Toxicology and Industrial Health,PMID: 22491721.http://dx. doi. org/10. 1177/0748233712442731
BadieiK,PourjaafarM andNowrooziaslA (2005).Effect of dried garlic powder(Allium sativum)on lead content of different tissue following subclinical lead poisoning in goats.IranJ.Vet.Res.,6(1): 12-16.

Bahramsoltani R, Farzaei MH, Rahimi R (2014). Medicinal plants and their natural components as future drugs for the treatment of burn wounds: an integrative review. Arch Dermatol Res., 306: 601-617.

Bancroft and Gamble(2002).Theory and Practice of Histological Techniques.5th ed., Churchill Livingstone, London, New York and Philadelphia.

BarbosaG (2006).Occupational exposure to toluene and its possible causative role in renal damage development in shoe workers.International Archives of Occupational and Environmental Health,79 (3):259-64.

Bedi PSand PriyankaS(2012).Effects of garlic against 7-12,dimethyl benzanthracene induced toxicity in Wistaralbino rats.Asian J Pharm Clin Res.,5 (4): 170-173. 
Block E. (1985).The chemistry of garlic and onions.Sci. Am.252:114-119.

BozinB,Dukic NM,SamojlikI,GoranA and IgicR(2008).Phenolics as antioxidants in garlic(Allium sativumLinn,Alliaceae).Food Chem,111: 925-929

Brozosky,Mand Winkler EG (1965).Glue sniffing in children and adolescents.NYState MedJ.,65:1984-1989.

Chang WJ, Joe KT, Park HY, Jeong JD, Lee DH (2013). The relationship of liver function tests to mixed exposure to Lead and organic solvents. Annals of Occupational and Environmental Medicine, 25:5.

de Oliveira HM, Dagostim GP, da Silva AM, Priscila Tavares P, da Rosa LAZC, and de Andrade VM (2011). Occupational risk assessment of paint industry workers. Indian J Occup Environ Med., 15(2): 52-58. doi: 10.4103/0019-5278.90374

Funada,M.,Sato,M.,Makino,Y.,Wada,K.,2002.Evalu ationofrewarding effect of toluene by the conditioned place preference procedure in mice. Brain Res. Protoc.10: 47-54.

Gedik N,Kabasakal Land Sehirli O (2005).Longterm administration of aqueous garlic extract (AGE)alleviates liver fibrosis and oxidative damage induced by biliaryobstruction in rats. Life Sci., 76:2593606.

\section{Lee}

Gerasimov MR, Collier L, Ferrieri A, Alexoff D,

GiffordAN,Balster,R.L.(2003).Tolueneinhalationproducesa conditionedplacepref- erenceinrats.Eur J Pharmacol., 477: 45-52.

Gerasimov MR, Schiffer WK, Marstellar D, Ferrieri R, Alexoff D, Dewey SL (2002). Toluene inhalation produces regionally specific Changes in extracellular dopamine. Drug Alcohol Depend. 65:243-251.

Gonzalez-YebraAL,KornhauserC,WrobelK,PerezLuqueEL,WrobelK,and Barbosa G (2006). Occupational exposure to toluene and its possible causative role in renal damage development in shoe workers. International Archives of Occupational and Environmental Health, 79 (3):259-264.

\section{Gorinstein S,Drzewiecki}

H,Leontowicz M,Najman K,and Jastrzebski Z.(2005).Comparison of the bioactive compounds and antioxidant potentials of fresh and cooked Polish,Ukrainian,and Israeli garlic.J Agric Food Chem,53: 2726-2732.

GospeSMand CalabanMJ (1988).Central nervous system distribution of inhaled toluene.Fundamental and applied toxicology,11: 540-545.

Gotohda T, Nishimura A and Morita K. (2009).Immunohistochemical studies on early stage of hepatic damage induced by subacute inhalation of toluene vapor in rats. J AppliedToxicol., 29(6): 505-509.

GuzelianP,MillsSand FallonHJ (1988).Liver structure and function in print workers exposed to toluene.JOccupMed.,30: 791-796.

Hassan HA,Hafez HS and Zeghebar FE(2010).Garlic oil as a modulating agent for oxidative stress and neurotoxicity induced by sodium nitrite in male albino rats.Food and Chemical Toxicology, 48: 1980-1985.
Henry RJ (1974). Clinical Chemistry.Principles and Techniques. 2nd ed. Hagerstown, MD, USA: Harper and Row.

Himnan,D.,1984.Tolerance and reverse tolerance to toluene inhalation: Effects on open-fieldbehavior. Pharmacol.Biochem.Behav. 21: 625-631.

HudakA,and UngvaryG (1978).Embryotoxic effects of benzene and its methyl derivatives: toluene,xylene.Toxicology,11: 55-63.

Hussein JS,Oraby FS andEI-Shafey N (2007).Antihepatotoxic effect of garlic and onion oils on ethanol induced liver injury in rats.JApplSciRes.,3(11): 1527-1533.

HusseinA,AbdallaM,HusseinJ,ShoushaW andMohamedA (2008).Antioxidants in shoe-makers exposed to organic solvents.Journal of Applied Science and Research,4:1107-1117.

Jalali FSS, Tajik H, Javedi S, Mohammadi BH, Athari SSA et al. (2009).The efficacy of alcoholic extract of garlic on the healing process of experimental burn wound in the rabbit. Journal of animal and veterinary advances, 8(4): 655-659.

Khan AA, Sultan R, Zamani GY and Ur-Rahman S (2013).Biochemical and hematological Analysis after exposure to hazardous materials during Shoe making. Journal of Biology and Life Science, 4(2): 116-138. www.macrothink.org/jbls

Killeen, C., Meehan, T., Dohnal, J., \&Leikin, J. B. (2011).Pseudorenal insufficiency with isopropyl alcohol ingestion. American journal of therapeutics,18(4), e113e116.

Kim H (2010). Occupational diseases in workers exposed to organic solvents. Hanyang medical reviews, 30(4):313-318.

KinghtA,PawscyC,AroneyR,LawrenceJR,JonesDB,a nd NewlandRC (1991).Upholsteres glue associated with myocarditis,hepatitis,acute renal failure and lymphoma.The Medical Journal of Australia,154 (5): 360-362.

KoderaY,SuzukiA,ImadaO,KasugaS,SumiokaI,

KazenawaA,TaruN,FujikawaM,NagaeS,MasamotoK,

Maeshige $\mathrm{K}$ and $\mathrm{OnoK}(2002)$.Physical,chemical,and biological properties of S-allylcysteine,an amino acid derived from garlic.JAgricFood Chem.,50: 622-632.

Lee DE, Gerasimov MR, Schiffer WK, and Gifford AN (2006). Concentration-dependent conditioned place preference to inhaled toluene vapors in rats. Drug and alcohol dependence, 85(1): 87-90.

Li D, Han Y, Meng X, Sun X, Yu Q, Li Y, Wan LHuo Y andGuo C (2010). Effectof regular organic solvents on cytochrome P450-mediated metabolic activities in rat liver microsomes. The American Society for Pharmacology and Experimental Therapeutics, , 38(11): 1922-1925.

LindemannR (1991).Congenital renal tubular dysfunction associated with maternal sniffing of organic solvents.Actapaediatricascandinavica,80: 882-884.

Londhe VP, Gavasane AT, Nipate SS, Bandawane DD andChaudhari PD (2011). Role of garlic (Allium 
sativum) in various disease: An overview. Journal of Pharmaceutical Research And Opinion, 1(4):129-134.

LowL K,Meeks JR and Mackerer CR(1988).Health effects of the alkyl benzenes.I.Toluene.Toxicol.Ind.Health,4:49-75.

MellertW,DeckardtK, Kaufmann Wand van RavenzwaayB (2007).Ethylbenzene: 4- and 13-week rat oral toxicity.Arch.Toxicol.,81: 361-370.

Mikail HG (2010): Phytochemical screening,elemental analysis and acute toxicity of aqueous extract of Allium sativum L.bulbs in experimental rabbits. JMedPlants Res.,4(4): 322-326.

Mirunalini S,Kumaraguruparan R,Subapriya R andNagini S(2004).Garlic oil enhances hepatic and blood antioxidants during hamster buccal pouch carcinogenesis.Pharmaceutical Biology,42(3):240-245.

Moser VC.,Balster RL (1981).The effects of acute and repeated toluene exposure on operant behavior in mice.NeurobehavToxicolTeratol. 3: 471-475.

MoszczyńskiP,andLisiewicz J

Hematological indices of peripheral blood in workers occupationally exposed to benzene, toluene and xylene. ZentralblBakteriolMikrobiolHyg B., 178(4): 329-39.

Moszczynski P andLisiewicz J (1985).Occupational exposure to benzene, toluene and xylene and the lymphocyte lysosomal N-acetyl-beta-dglucosaminidase. Ind Health. 23:47-51.

NawrotP,and Staples R (1979).Embryo-fetal toxicity and teratogenicity of benzene and toluene in the mouse.Teratology, 19:41.

Niaz K, Bahadar H, Maqbool F andAbdollahi M (2015). A review of environmental and occupational exposure to zylene and its health concerns. EXCLI J, 14:1167-1186.

ParafF,LewisJ andJothyS(1993).Acute fatty liver of pregnancy after exposure to toluene.Journal of clinical gastroenterology,17: 163-165.

Pari L,Murugavel P,Sitasawad SL and KumarKS(2007).Cytoprotective and antioxidant role ofdiallyltetrasulfide on cadmium induced renal injury: an in vivo and in vitro study.Life Sci.7:50-658.

Reitman S and Frankel S (1975).A colourimetric method of serum glutamic oxalo acetic and glutamic pyruvic transaminases.Am J ClinPathol. 28: 56-63.

Sarhan and Hussein (2016) Hazards effects induced by inhalation of isopropanol.Under in press.
Shaarawy SMTohamyAAandElgendySM(2009). Protective effects of garlic and silymarin on NDEAinduced rat's hepatotoxicity IntJBiolSci.,5(6): 549-557.

Sharma V, Sharma A and Kansal L (2010). The effect of oral administration of Alliumsativum extracts andlead nitrate induced toxicity in male mice. Food ChemToxicol., 48(3):928-936. doi: 10.1016/j.fct.2010.01.002. Epub 2010 Jan 12.

Streicher HZ,Gabow PA, Moss AH,Kono D andKaehny WD (1981). Syndromes of toluene sniffing in adults. Annals of Internal Medicine, 94(6): 758-62.

TasU,OgeturkM,MeydanS,KusI,KulogluT,IlhanN,K oseE,and SarsilmazM(2011).Hepatotoxic activity of toluene inhalation and protective role of melatonin.Toxicological andIndustrial Health,27(5): 46573.

Taofeeq O, Olayinka RG, Taiwo OO, Ganiyu AO, Dallatu Mohammed Kabiru DM, and Suleiman MA (2015). Organic solvent exposure: hepatotoxicity, nephrotoxicity, and haematotoxicity assessment amongst vehicle spray painters in Ile-Ife, Nigeria American Journal of Environmental Protection, 3(3): 95-99.

Vaghasia KK, Desai KR, George LB, and Highland HN (2013).Evidence of oxidative stress, biochemical and histological alterations in kidney and liver on short term inhalation of a specific mixture of organic solvents. International Journal of Pharmaceutical Science and Health Care, 6(3):113-130.

Von BurgR (1993).Toxicology update: toluene.J ApplToxicol.,13: 441-446. WafayH,El-SaeedG,

ToukhyS,YounessE,EllaithyN,AgaibiM andEldalyS (2012).Potential effect of garlic oil and silymarinon carbon tetrachloride-induced liver injury.Australian Journal ofBasic and Applied Sciences, 6 (3): 409-414.

Wynn JG, Bird MI, Vellen L, Grand-Clement E, Carter J and Berry SL (2006). Continental-scale measurement of the soil organic carbon pool with climatic, edaphic, and biotic controls. Global Biogeochemical Cycles, 20(1): GB1007, doi:10.1029/2005GB002576.

Zaman F, Pervez A, \&Abreo K (2002). Isopropyl alcohol intoxication: a diagnostic challenge. American Journal of Kidney Diseases, 40(3): e12-1. 